\title{
¿Qué significa aprender? Preguntar, pensar, aprender desde la mirada de filosofía para niños
}

Pablo Flores del Rosario Doctor en Filosofía de la Ciencia Instituto Superior de Ciencias de la Educación del Estado de México pablofloresdelrosario@hotmail.com https://orcid.org/0000-0003-0808-9364

\section{Artículo de investigación}

Recepción: 15 de enero de 2019 Aprobación: 09 de marzo de 2019 https://doi.org/10.19053/22160159.v10.n23.2019.9689

\section{Resumen}

El objetivo del trabajo consiste en argumentar la tesis de que se aprende pensando y se piensa cuando se pregunta, en contra de la tesis neoliberal de aprender haciendo. De esta argumentación se infiere un concepto de educación fundado en la complejidad. Se trabajó la metodología de Filosofía para Niños $\mathrm{y}$, para interpretar los datos y analizar lo que discutieron los niños, se hizo un ejercicio de carácter hermenéutico. Se trabajaron temas como las habilidades de indagación, formas de relación que demuestran lo actitudinal, saber argumentar y exponer resultados. Aquí se expone una sesión que posibilitó presentar con detalle los resultados de ese ejercicio metodológico.

Palabras clave: filosofía, niñez, currículo formal, habilidades de investigación, hacer 


\section{What does it mean to learn? Asking questions, thinking, learning from the point of view of philosophy for children}

\section{Abstract}

The purpose of this work is to support the thesis that we learn by thinking and we think when we ask questions, as opposed to the neoliberal thesis of learning by doing. A concept of education based on complexity is implied from this argumentation. Philosophy for children was used as a work methodology. A hermeneutic exercise was carried out to interpret data and to analyze what children were discussing. Issues such as questioning skills, forms of relationships that show attitudes, knowing how to argue and how to present results were addressed. This text presents a session which allowed for a detailed presentation of the results of this exercise.

Keywords: philosophy, childhood, formal curriculum, questioning skills, to make

\section{Quelle est la signification d'apprendre? Poser des questions, penser, apprendre du point de vue de la philosophie pour enfants}

\section{Résumé}

L'objectif de ce travail est de soutenir la thèse que l'on apprend en pensant et on pense lorsque l'on pose des questions, contrairement à la thèse néolibérale d'apprentissage par la pratique. Il s'en suit un concept d'éducation fondé sur la complexité. La méthode de travail employée est 
la philosophie pour enfants. Un exercice de nature herméneutique a été réalisé pour interpréter les données et analyser ce qui a été discuté par les enfants. Des sujets tels que les compétences en matière d'interrogation, les formes de relation montrant les attitudes, le fait de savoir comment argumenter et comment exposer des résultats ont été abordés. Ce texte présente une séance qui a permis de montrer les résultats de cet exercice méthodologique en détail.

Mots-clés : philosophie, enfance, programme formel, compétences d'interrogation, faire

\section{O que significa aprender? Perguntar, pensar, aprender desde a olhada da filosofia para crianças}

\section{Resumo}

O objetivo do trabalho é argumentar a tese de que se aprende pensando e se pensa quando se pergunta, em contra da tese neoliberal de aprender fazendo. Deste argumento infere-se um conceito de educação baseado na complexidade. Foi trabalhada a metodologia de Filosofia para as Crianças e, para interpretar os dados e analisar o que as crianças discutiram, se fez um exercício de caráter hermenêutico. Foram trabalhados temas como habilidades de indagação, formas de relação que demonstram a atitudinal, saber como argumentar e expor os resultados. Aqui se expõe uma sessão que possibilitou apresentar em detalhe os resultados desse exercício metodológico.

Palavras-chave: filosofia, infância, currículo formal, habilidades de pesquisa, fazer 


\section{Introducción}

La investigación es de naturaleza cualitativa, al partir del análisis de las acciones ocurridas en el salón, en un grupo de sexto grado de educación primaria. De ahí emergen varios supuestos que forman la trama que se articula. Para tener una idea de la perspectiva desde la que se discute el trabajo, se esbozan algunos de tales supuestos, que al final se hacen aserciones garantizadas de una idea de educación, que subyace al proyecto neoliberal. Un supuesto articulador es el que asume que la clase debe desplegarse a partir de un conjunto de actividades organizadas para que el estudiante las haga como parte de sus actividades de clase. Tales actividades se acomodan a los objetivos propuestos por el nuevo programa y la lectura que de este se hace. Las actividades implican la acción de los estudiantes, incluso en la misma fase de cierre, que con frecuencia consiste en un sumario o conclusión, que sintetiza los resultados. El supuesto implica el trabajo de los miembros del grupo, pero no se interesa por procesos que son de naturaleza colectiva: procesos inferenciales, cognitivos, emocionales, etc. Como puede verse, desde esta perspectiva el centro no es el estudiante, sino el proceso centrado en un conjunto de actividades, cuyo supuesto de base es que despliega los procesos mencionados, pero de ellos no se interesa. $\mathrm{Al}$ no interesarse en ellos, inhibe su despliegue conscientemente realizado.

En consecuencia, este trabajo expone tres premisas de la práctica con Filosofía para Niños [FpN]: los niños desarrollaron sistemáticamente y conscientemente habilidades para investigar; los niños trabajaron de modo comunitario y no de modo aislado como es habitual en este grupo de sexto grado de educación básica; los niños discutieron y se apropiaron de tópicos que forman parte del currículo formal. Estas premisas permiten fundamentar la idea de que "Los niños aprenden pensando, no haciendo", que es la tesis de este trabajo. Será en las premisas donde pueden verse en movimiento tópicos como las habilidades de investigación, las formas de relación que despliegan lo actitudinal, el saber argumentar y exponer resultados, que incluso forma parte de lo que se llama perfil de egreso, entre otros más puntuales como los contenidos del programa. Esto es posible porque la práctica con FpN permite inferir un despliegue consciente y sistemático de esas habilidades. 
El objetivo del trabajo consiste en argumentar la tesis de que se aprende pensando, contra la tesis neoliberal de aprender haciendo. De esta argumentación se infiere un concepto de educación fundado en la complejidad. Si bien es cierto que se trabajó la metodología de FpN, también, para interpretar los datos que arrojó ese trabajo, se hizo uso de un ejercicio metodológico de carácter hermenéutico, cuyo resultado fue la tesis antes anotada y que se explaya en el trabajo. Aquí se expone una sesión que posibilitó presentar con detalle los resultados de ese ejercicio metodológico.

Cabe aclarar que se trabajó con un grupo de sexto grado de educación primaria. El contexto es rural, aunque hay mucha cercanía con la ciudad de Toluca, en el Estado de México. La misma naturaleza del contexto hizo difícil iniciar el trabajo de FpN, pues los niños de la comunidad no tienen mucho espacio para ejercer el derecho al habla, menos para hacer preguntas durante la clase y discutir sus posibles respuestas. Pero sí hacían ejercicios donde, dadas las preguntas, debían pasar al frente para leer sus respuestas. Así que una comunidad de indagación fuealgo novedoso, a lo que poco a poco fueron habituándose. La sesión que sirve para exponer los resultados fue trabajada al final del semestre, que coincidió con el fin de la investigación.

\section{Una comunidad de diálogo que provoca la emergencia del pensar}

Filosofar con niños es posible en la medida en que se construya con ellos un espacio que haga pertinente la exigencia del pensar. Construir este espacio implica movimientos, no siempre previstos, porque pensar y las condiciones para hacerlo son siempre imprevisibles. A pesar de esta indicación, en $\mathrm{FpN}$ se ha trazado una ruta metodológica que permite acotar esa imprevisibilidad. Sin embargo, a lo largo del camino se puede seguir esta ruta o modificarla. Consiste en varias fases y elementos en cada una de ellas. A continuación, hacemos su descripción, tal como fue diseñada en esta investigación.

\section{Fase de apertura.}

Son las normas que guiarán la interacción en el aula. Estas normas surgen de los participantes, bajo la inicial convicción de interactuar 
haciendo que se sientan cómodos, incluidos y seguros durante el trabajo en comunidad de diálogo. Esta convicción inicial permite que los miembros del grupo propongan diversas normas, que al ser propuestas por cada uno les harán habitable la hora de actividad. La continuidad en el uso de normas para interactuar en la clase, poco a poco se incorporan como hábitos para actuar, incluso fuera de la hora de esa actividad.

\section{Fase de desarrollo.}

Se compone de cuatro elementos. El primero consistió en la lectura del cuarto apartado, del capítulo 1, de Pixie (Lipman, 1998, p. 6-8), del currículo de FpN, previamente leída y acotada en sus posibles rutas de discusión y habilidades para indagar que podrían emerger - claro que cuando se tiene dominio del programa, se puede iniciar con otros recursos: una pintura, un dibujo, una frase, baile, etc.-. La idea es enfocar el grupo hacia algún punto común que les cause perplejidad, que les lleve a maravillarse o, al menos, que les permita tener un punto de vista diferente sobre algún hecho o situación de su vida, que después se fragmentará en diversos puntos bajo la lógica de una diversidad de preguntas. En este caso se leyó ese segundo apartado. La lectura fue por turnos y en voz alta. El segundo momento consistió en solicitarles una pregunta que les haya sugerido lo que leyeron. Esa solicitud se articuló con estas preguntas: ¿qué pensaron? ¿Podrían hacer de eso que pensaron una pregunta? ¿Qué pregunta podría ser?; y con esta indicación: iEscriban su pregunta por favor! Cada niño escribió su pregunta, pero, como el grupo era numeroso - cuarenta niños-, se pidió que elaboraran preguntas por equipo de trabajo. Aquí los niños iniciaron, en cada equipo, el diálogo en torno a sus preguntas, pues debían formular una pregunta en ese equipo. El tercer punto consiste en la construcción de una agenda de discusión. Con las preguntas que cada equipo elaboró, se pidió que cada pregunta, escrita en hoja de papel, tuviera el nombre del equipo, como una forma de responsabilizarse por lo que se dice o se pregunta. Aquí se continúa la discusión en torno a cuáles preguntas son más abarcadoras que otras o cuál pregunta podría sintetizar lo que expresan las otras. El cuarto es el paso a la discusión de las preguntas que la comunidad de diálogo ha argumentado como las viables para trabajar, además de continuar con el modelo de preguntas anotadas antes. Sugiero 
introducir preguntas que impliquen habilidades de indagación en la escuela. Lipman plantea como necesidad, distinguir "las destrezas esenciales para indagar en un entorno escolar [...] Esto me ha llevado a hablar sobre cuatro tipos de destrezas cognitivas" (2004, p. 55): investigación, razonamiento, formación de concepto y traducción. Cada destreza implica preguntas pertinentes. Más puntualmente, en Splitter \& Sharp, se sugiere una lista de preguntas "que alienta la indagación" (1996, pp. 84-85), que apunta a varias fases del proceso de la comunidad de indagación, usadas por nosotros en las sesiones respectivas.

\section{Fase de cierre.}

Se compone de dos elementos: el cierre de la sesión y su evaluación. El cierre de la sesión, se perfila cuando la comunidad llega a la clarificación de la pregunta o a una posible respuesta, previo movimiento de las habilidades de indagación del currículo de FpN -que puede verse de modo esquemático en el libro de Echeverría (2004, pp. 232-238) - ahora como preguntas, como se ha anotado antes. En Splitter \& Sharp (1996, pp. 25-26) se ofrece una lista de estrategias que pueden ser identificadas bajo el título pensar, esto es, una lista de esas habilidades. El cierre es la parte conclusiva de la discusión.

\section{Fase de evaluación.}

Con dos elementos, la evaluación de las normas aceptadas por la comunidad y la evaluación de posibles aprendizajes, como producto de la discusión. Dependiendo del nivel educativo, se podrán implementar diversos formatos de evaluación. Como puede verse, se sigue la idea plantada sobre educación basada en habilidades para la vida, desarrollada por las agencias de Naciones Unidas (Portillo-Torres, 2017, p. 3).

Cuando se inició la investigación, los alumnos no hablaban, a menos que las preguntas fueran cerradas - con respuesta: sí o no-. Aquí fue necesario desplegar las preguntas más pertinentes para trabajar las habilidades de indagación, que son indispensables en una comunidad de diálogo, y en general, en el proceso enseñanzaaprendizaje. Esto es lo normal en el proceso de esta nueva forma 
de trabajo, que no es un trabajo con dinámicas grupales o en equipos. Tiene su propia dinámica, que está articulada por el trabajo en comunidad, el diálogo y la lógica de las preguntas. Esta dinámica rompe con el modelo que privilegia el hacer como factor determinante del aprendizaje.

Quienes participan en una comunidad de diálogo se abren a la lógica de las preguntas. Esas preguntas se van incorporando como hábitos entre los participantes, hasta que ellos mismos terminan por plantearlas, así que quien facilita esta tarea poco a poco se siente inmerso, como participante, en la misma tarea que ha facilitado. Desaparece la figura del profesor para perfilarse una auténtica comunidad de diálogo. Esa es la idea, convertir el salón de clase en una comunidad de diálogo madura (Splitter \& Sharp, 1996, p. 203), cuestión que se logró con este grupo, como se verá enseguida.

\section{Aprender pensando desde la comunidad de investigación}

La sesión de FpN es algo que los niños deben construir. De este modo, se les sugiere que diseñen un espacio que sea su espacio, que en consecuencia será un espacio donde las normas serán formuladas por ellos mismos, bajo el supuesto de que mantener relaciones de grupo desde normas propuestas por el grupo mismo implica el compromiso con su respeto. Para provocar esto, se planteó la pregunta: ¿Qué normas se imaginan que puedan hacer posible un lugar donde trabajen con mucho gusto? Estas fueron algunas normas que el grupo propuso:

1. No jugar mientras el grupo trabaje.

2. No hacer ruido durante el trabajo.

3. Participar en el trabajo.

4. Tomar turnos.

5. Escuchar a sus compañeros.

6. Hacer preguntas.

Después iniciamos el trabajo con una lectura de Pixie, cap. 1 (Lipman, 1998). Esta lectura lleva a una serie de preguntas que los niños plantean a la comunidad. Las preguntas surgen de la indicación del coordinador de la sesión: ¿qué les hizo pensar lo que leyeron? ¿Pueden hacer una pregunta de lo que pensaron? 
Cada niño se dio a la tarea de hacer su pregunta. Dado que era un grupo numeroso, 40 niños, se hizo una división para trabajar las preguntas en grupo. Las indicaciones fueron: "(I) formen grupos de trabajo de cinco niños, (II) escriba cada uno una pregunta de lo que les haya sugerido la lectura que acaban de hacer, (III) discutan sus preguntas y seleccionen una para que la escriban en su hoja”. Los niños discutieron sus preguntas en cada grupo. Después discutieron la pregunta que resultaba pertinente, porque representaba la pregunta de cada uno. Las preguntas seleccionadas fueron las siguientes:

1. ¿Por qué los niños tienen miedo?

2. ¿Por qué a veces se duerme un brazo?

3. ¿Por qué a la niña se le durmió su brazo?

4. ¿Por qué tuvo miedo al sentir el brazo dormido?

5. ¿Sentimos miedo cuando estamos dormidos?

6. ¿Tenemos miedo cuando estamos despiertos?

7. ¿Qué es lo que nos causa más miedo?

8. ¿Podemos tenerle miedo a los padres o a los maestros?

Este primer movimiento, objetivado en las ocho preguntas de los equipos, que parece elemental, implica el movimiento de varias categorías del pensar, como investigar, razonar, conceptualizar, traducir y mantener la disposición crítica (Lipman, 2004), mente abierta y dispuesta a lo maravilloso, que entre los niños aparece con frecuencia. Así, hacer una pregunta que nos sugiera algo que nos llama la atención, como se solicitó en la indicación II -en este caso se trató de una parte de la lectura que tiene que ver con algo personalmente pensado o vivido; en otros casos con algo que nos llama la atención de algún paisaje de la realidad-implica estar abierto al proceso de maravillarnos - apertura a interrogar eso que nos maravilla- y a buscar alguna respuesta a nuestro interrogante, lo que nos sitúa en el terreno de la investigación. Esto es lo que pasó en las ocho preguntas elaboradas y en cada niño que participó.

Hacer una pregunta y hacer una pregunta de lo que nos genera perplejidad es lo que traza la diferencia en la concepción de aprendizaje. En el primer caso, hacer una pregunta, lleva a la mera emisión de un enunciado constatativo - solo se quiere, en esa pregunta, constatar lo que ya se sabe-. Hacemos, preguntamos, lo que ya sabemos. En el segundo caso, nos apropiamos, al tener una experiencia, del objeto que nos ha maravillado y lo hacemos 
al interrogarlo y fragmentarlo en sus componentes conceptuales. Eso fue lo que hicieron los niños. Les maravilló leer algo que ellos habían vivido, pero sobre lo que no se habían preguntado. Solo nos deja perplejos lo que nos confronta con lo que sabemos, esto es, con nuestras evidencias inmediatas. En esta confrontación, lo que sabemos se desarma, no tiene componentes para entenderlo, no puede entenderlo sin antes acomodarlo de algún modo. El modo de hacerlo, para los niños, fueron sus preguntas. Como implicación, viene un trabajo conceptual que hace posible pensar de otro modo - para no someter a nuestra inmediatez- el objeto de nuestra perplejidad. La primera consecuencia que se puede inferir de lo que hicieron los niños es: solo aprendemos cuando pensamos y solo pensamos al preguntar.

Pero entre lo que les llamó la atención a los niños, lo que los atrapó en sus redes, y sus preguntas hay una tensión que solo tiene solución en un proceso inferencial, que termina siendo una serie de cadenas inferenciales - esto es pensar-, porque entre las premisas y la conclusión, para que no resulten abstracciones de lo real, se mantiene otra tensión cuya solución está determinada por el uso de analogías, metáforas y modelos, que son partes del pensar (Lipman, 2016) y con ellas jugaron los niños en el proceso de discusión de sus preguntas. Razonar e investigar implica el uso de conceptos no siempre claros en los niños, pero siempre con un horizonte abierto a nuevos conceptos. Dicha apertura está posibilitada por sus ejercicios de traducción, que implican el uso de semejanzas, de la relación partes-todo, causa efecto (Lipman, 1998). Ello ocurre en el movimiento de estas categorías, que forman parte del pensar de los niños al elaborar sus preguntas.

Incluso esto puede verse cuando los niños discuten en grupo cuál pregunta seleccionarán. En un equipo de trabajo los niños sostienen la siguiente discusión:

(N) "Si elegimos alguna de nuestras preguntas no estaría bien porque todas son importantes".

(J) "Cierto, todas son importantes porque dicen algo que nos gusta”.

(P) "Sí, pero aunque nuestras preguntas sean importantes por decir lo que nos gusta, podemos hacer una lista y ordenarlas".

(T) “¿Ordenarlas cuando todas son importantes?”. 
(Pa) "Eso dijeron".

(C), "Bueno, piden una sola pregunta, por eso hay que ordenarlas".

(T), “¿No estamos preguntando todos lo mismo?”.

(I), "Tal vez sí, tal vez, pero de diferentes cosas o de diferentes maneras".

Preguntar lo mismo, pero de diferentes cosas o de diferentes maneras, cuando todos preguntan lo mismo, implica un modo de pensar que ya pone en juego diferentes categorías: trazar distinciones y al mismo tiempo buscar semejanzas, ubicar la relación partetodo, causa-efecto. El juego de estas categorías permite que los niños puedan tomar una decisión fundada en razones, evidencias e inferencias pertinentes. Desde aquí, desde este juego construyen una relación con la idea de indagación en el aula.

Poner en juego esta serie de categorías del pensar, como se puede notar en el diálogo anterior, llevó al grupo a plasmar la pregunta con la que se trabajó la sesión. La pregunta aparece sin interrogantes, pese a ser una pregunta. Sintácticamente por la ausencia de los signos de interrogación no hay pregunta alguna, tan solo una afirmación. Para los niños, resultó claro que había una pregunta en lo escrito en el pizarrón: "Qué es el miedo" -¿Qué es el miedo?-. Pregunta resaltada en medio de otras preguntas:

¿Qué es el miedo?

¿Qué es lo que nos causa más miedo?

¿Podemos tenerles miedo a los padres o a los maestros?

Llegar a esta pregunta implicó, para los niños, varios movimientos del pensar. Los niños discriminaron y trazaron distinciones entre las preguntas. Pero trazar una distinción entre una serie de preguntas es posible en la medida en que se aplica el principio de semejanza y diferencia. Hacerlo implica usar analogías y metáforas (Lipman, 2016). La serie de preguntas que guían este proceso permite que los niños tomen la decisión de trabajar una pregunta y no otra. Como puede verse, de las preguntas de los niños se hace un punto de partida para elaborar más preguntas que permitan perfilar cierta decisión. Pero el perfil es determinado por los niños que participan, en la medida en que la respuesta a las preguntas será una respuesta que parte de la ruptura de su mundo de vida. Esta ruptura es notoria en las preguntas que hicieron. 
Bachelard anotaba que "la evidencia primera no es una verdad primordial” (1953, p. 11), porque exigía que para lograr la objetividad científica se debía romper, en primer lugar, con el objeto inmediato, lo que se logra cuando contradecimos los pensamientos que nacen de la observación primera, desnuda. Por eso anotamos que las respuestas dadas por los niños implican la ruptura de su mundo de vida, de su mundo inmediato. En el mismo sentido, Bachelard anota que, lejos de maravillarse, el pensamiento debe ironizar Bachelard (1953, p. 11). Los niños lo hacen, porque, en efecto, parten de ese gesto de perplejidad que produce el maravillarse ante el mundo, mas no permanecen en la red generada por la perplejidad: rompen con ella. La forma irónica que adopta el pensamiento infantil consiste en construir otros conceptos para entender aquello que les causa perplejidad.

Del lado de las respuestas a la pregunta anotada, estas van desde la emisión de una sola palabra: "fantasmas", "animales", o dos palabras o más: "animales raros". Lo que denota un incipiente inicio del trabajo en comunidad. Poco a poco, en la misma sesión, las respuestas se van matizando y ampliando: “Todos debemos superar el miedo, por ejemplo al escalar la montaña. La gente sí tiene miedo a caer, pero tiene que superarlo al subir hasta la cima para superar ese miedo". De modo que los niños terminan por discutir la relación entre el miedo y el cuerpo humano, hasta expresar dos enunciados de carácter conclusivo: "existen reacciones del cuerpo ante el miedo"; "podemos decir que el corazón reacciona ante el miedo".

Ambas conclusiones implicaron la discusión del cuerpo humano, como parte de los seres vivos, la relación con su medio ambiente y su interacción con otros seres vivos, que nos llevó directo al contenido del Plan de Estudios de Educación Básica, cuyo objetivo es: "los estudiantes se aproximan al estudio de los fenómenos de la naturaleza y de su vida personal de manera gradual y con explicaciones metódicas y complejas, y buscan construir habilidades y actitudes positivas asociadas a la ciencia" (SEP, 2011, p. 51).

Pero además de discutir un tema de un plan de estudios discusión que siguió matizando la ruptura de los saberes previos de los niños-, al mismo tiempo se abrían nuevas perspectivas en cada uno de los participantes, y esto es aprendizaje. Estos aprendizajes también forman parte de los objetivos de un plan de estudios. 
"El aprendizaje no es lo que leemos, sino lo que resulta de la discusión crítica de lo leído"; esta frase de Feyerabend (1985, pp. 68-69) ilustra lo que queremos desarrollar en esta parte. Pero hay que aclarar que leer no es necesariamente asunto de libros. Ya en el Renacimiento se hablaba del "libro de la naturaleza". Leer entonces se toma en su acepción amplia. Aceptar la tesis de Feyerabend implica asumir que todo proceso de diálogo es un proceso de aprendizaje. Lo es en la medida que ante cada perplejidad vienen las preguntas que nos llevan a pensar y su resultado es el aprendizaje. Los niños, en efecto, no solo leyeron, también discutieron lo que leyeron cuando hicieron su agenda de preguntas. El aprendizaje producido por la discusión ha implicado aprender conceptos, procedimientos y actitudes. Todo enmarcado en las cinco categorías del pensar: investigación, razonamiento, formación de conceptos, traducción y disposición crítica. De este modo, los niños pueden aprender no solo cómo desplegaron sus procedimientos, sino qué habilidades de indagación pusieron en juego, todo realizado en grupos de trabajo y en discusión grupal.

A partir de esta lectura de una sesión de comunidad de investigación, se pueden hacer varias inferencias, como la de que el aprendizaje no es lo que hacemos, sino lo que hacemos cuando hemos pensado cómo hacerlo, porque hacer es simplemente continuar en nuestro entorno inmediato, adecuarnos al entorno. Hacer es una actividad, que cuando es repetida termina por hacerse mecánica, pero cuando mecanizamos una actividad dejamos de pensar, dejamos de poner en movimiento lo que sabemos, dejamos de preguntar. Pero, aprender implica el movimiento del pensamiento a partir de las preguntas.

"Dame una palanca y moveré el mundo"; es una frase de Arquímedes (1988) que implicó el desarrollo de un modo de pensar las relaciones entre las matemáticas y el mundo. Como el mismo Arquímedes lo expresa en una carta a Eratóstenes: "he creído oportuno confiarte por escrito, y explicar en este mismo libro, las características propias de un método según el cual será posible abordar la investigación de ciertas cuestiones matemáticas por medio de la mecánica" (1988, p. 35). Desde este nuevo modo de pensar es que adquiere sentido la frase indicada. Pero este modo de pensar es hecho contra un hacer inmediato.

El modelo de educación neoliberal asume una persistente inferencia que iguala los objetivos conductuales de Bloom (1971) con 
la idea de competencia. Esta inferencia persiste porque, a pesar de matizar las diferencias entre la taxonomía por objetivos de Bloom (1971) y las competencias (Eurydice, 2002), se sigue insistiendo en que ambas son lo mismo. Cuestión que se matiza en la observación repetidamente hecha entre los profesores: "al parecer la nueva propuesta curricular de trabajar con competencias consiste en dejar que el alumno haga sus tareas, pues al hacer se supone que aprende".

El proyecto curricular de enseñanza basado en competencias es un modelo que está teniendo aplicación en muchos países y no por ello es la panacea a los problemas educativos que esos países enfrentan. Cierto, no es la panacea del problema educativo en México, pero desde determinada perspectiva es lo que mejor acota los múltiples problemas que enfrentamos en este rubro, como el problema de los bajos porcentajes en los aprendizajes de las áreas evaluadas por PISA (OCDE, 2006).

Contra esta serie de tesis, producto del proyecto educativo neoliberal, sostenemos tesis contrarias, a partir de lo que nos ofrece la experiencia con $\mathrm{FpN}$, en particular en la sesión antes expuesta. $\mathrm{Si}$ aceptamos que la epistemología está relacionada con asuntos como el amplio rango de esfuerzos para conocer el mundo -incluyendo las no refinadas prácticas de la vida cotidiana del profano, así como los refinados métodos especializados del científico e investigador-, entonces habrá que suponer que en cada aprendizaje ponemos en juego este amplio rango de esfuerzos (Goldman, 1986). Esta definición se puede resumir en la tesis negativa: "no se aprende solo haciendo"; y en la tesis positiva: "solo se aprende poniendo en juego un amplio rango de esfuerzos". Justo esto es lo central de la propuesta de aprendizaje en el nuevo currículum. Aquí enlaza FpN, pues mientras allí se habla de rango de esfuerzos, aquí se habla de categorías del pensar. Este pensar consiste en ir "más allá de lo superficial”(Mejía, López, \& Valenzuela, 2015, p. 152).

Veamos algunos ejemplos de cómo esto aparece en la sesión de $\mathrm{FpN}$, descrita antes: leer y escribir, como aprendizajes, solo son posibles como logro si sostenemos una práctica cotidiana, que en el trabajo de FpN aparece cuando se lee y cuando se escriben las preguntas. Lo mismo ocurre en la exigencia de emplear la argumentación y el razonamiento al analizar situaciones, identificar problemas, formular preguntas, emitir juicios y proponer diversas soluciones, que pueden verse en lo que hicieron los niños: 
- Buscar, evaluar y sistematizar información.

- Pensar, reflexionar, argumentar y expresar juicios críticos.

- Analizar, sintetizar y utilizar información.

- Conocer y manejar distintas lógicas de construcción del conocimiento en diversas disciplinas y en los distintos ámbitos culturales, cuestión que se ve en el paso de la discusión al trabajo sobre el cuerpo humano, pues notaron que aquí se trataba de una búsqueda y tratamiento de información diferente.

- Relacionar armónicamente con otros y con la naturaleza.

- Comunicar con eficacia.

- Trabajar en equipo, hacer acuerdos y negociar con otros.

- Crecer con los demás.

- Manejar armónicamente las relaciones personales y emocionales, que aparece como parte de las normas, que les permite un espacio de convivencia y aprendizaje.

- Conocer y valorar sus características y potencialidades como ser humano, que le permite identificarse como parte de un grupo social, emprender proyectos personales, esforzarse por lograr sus propósitos y asumir con responsabilidad las consecuencias de sus acciones, ciertamente este aspecto aparece como algo marginal, pero podría trabajarse en siguientes sesiones.

Recordemos la tesis básica de este trabajo: no se aprende haciendo. Esto es: “No se trata que los estudiantes 'hagan' sus actividades sin mayor trámite, no quiere decir repartir la tarea para que la 'hagan'. Ese hacer implica la intervención de habilidades de pensamiento y de "mediadores"' (Vygotski, 2008).

En el fondo se trata de enfrentar dos problemas asociados. El primero consiste en trazar la distinción entre hacer alguna actividad y hacerla siguiendo determinadas estrategias y reglas, que implicarían la construcción de un problema, de una paradoja, de un dilema o de una pregunta. El segundo consiste en comprender que una actividad despliega determinadas habilidades de indagación en la medida en que se guían por estrategias y reglas coordinadas. Solo en esta distinción se puede entender que las tareas asignadas movilizarán todos los recursos de los niños. Solo cuando se construye un problema se pueden trazar estrategias de solución. Filosofar con los niños ayuda a la realización de estas tareas e impacta en lo que podríamos llamar un aprendizaje. 
Un aprendizaje se logra cuando en un diálogo -que se ubica en una comunidad de investigación- los participantes logran tener claro el lugar donde la pregunta toma sentido, o donde las palabras y los conceptos que componen la pregunta, el problema o la paradoja toman su sentido. Como en el caso de estos niños, que concluyen: "quizá la palabra miedo nos la inculquen, nos la digan con frecuencia, para que seamos respetuosos con los mayores, pero mientras pueden hacer que nos enfermemos".

La conclusión de los niños está articulada por su capacidad de diferenciar un problema en su componente social y biológico. Trazar diferencias y buscar semejanzas es algo que se hizo en las siguientes sesiones. Esta distinción entre hacer para aprender y pensar para aprender, inferida aquí, nos lleva a matizar otra idea de educación.

\section{La diseminación de una concepción de educación a partir de este ejercicio}

$\mathrm{Si}$, como se expondrá enseguida, el proyecto educativo neoliberal reduce el proceso educativo a tareas, tiempos, movimiento y productos - aprender haciendo -, entonces se debe desplegar un concepto de educación fundado en la complejidad, que permita entender la complejidad de los procesos de indagación en FpN. El mismo ejercicio anterior lo permite.

La educación se mueve en la incertidumbre, porque la relación educativa ocurre cuando al menos dos personas se encuentran. El grado de incertidumbre crece con el número de sujetos en ese encuentro, hasta volverse inmanejable. Nada puede pre-verse como condiciones o resultados del encuentro. Si se torna inmanejable podría hacerse algo difuso, lo que obliga a convertir esa relación en un diagrama. Ahí se sitúa una serie de dispositivos para el dominio de esa incertidumbre inmanejable. A esos dispositivos se les categorizará como proceso educativo. Contra esta idea debemos entender que la relación educativa es el mismo encuentro que se va tejiendo con la mirada y la palabra. Aunque a veces ni la mirada ni la palabra alcanzan para trenzar el encuentro. Entonces participamos de cuerpo entero: gestos y movimientos se acompañan de miradas y tonos de voz, que por ser manifestación de la singularidad de cada participante permiten asegurar la relación educativa. El diálogo en 
acción, FpN, teje relaciones para hacer manejable la complejidad del acto educativo. Esas relaciones, que esclarecen esa incertidumbre, están fundamentadas en la lógica de las preguntas, que es lo que hace posible el pensar, que implica niveles como la razonabilidad, el buen juicio y la capacidad de apreciar (Lipman, 2016, p. 23). Las preguntas esclarecen la incertidumbre de todos y cada uno - se va haciendo más clara en la medida que esas preguntas permiten que todos hagan el ejercicio del pensar-.

Como ha enseñado el psicoanálisis, aunque su génesis sea producto de entender la represión sexual de su época (Roudinesco, 2018), no todo lo que ocurre en cualquier encuentro es consciente. El inconsciente mismo participa del logro del encuentro, porque el deseo es lo que permite o no esa relación. Entonces el problema de la educación se torna complejo, porque para que ocurra debe ocurrir el encuentro, que no consiste simplemente en que dos personas se encuentren en algún tramo de su vida, porque debe estar presente el deseo para que ocurra cualquier relación de encuentro. Sin el deseo solo ocurre el encuentro de dos cuerpos que pasan de largo, dos cuerpos que se cruzan y no se afectan. Ocurre el encuentro, pero ninguna relación - menos la relación educativa - Esta relación de cuerpos que se encuentran y pasan uno junto al otro no podría llamarse relación, porque ella implica la afección. Solo hay relación si hay choque de cuerpos: los gestos, las miradas, el lenguaje, las preguntas y el pensar lo determinan -ahí se instala la relación educativa, cuyo movimiento inicia cuando se ponen a funcionar los tres radiales, que son facetas de la filosofía, en tanto las tres son formas de investigación (Lipman, 2004, p. 112)-.

Toda relación educativa es posible en el encuentro y afección, entre al menos dos cuerpos. Pero el mismo encuentro es producto del deseo si queremos una relación educativa. Aquí el deseo se convierte en algo productivo - y no lo que nos falta - , cuando se introduce el diálogo como garante y esclarecedor de ese encuentro. Cierto, la educación, como relación o como acción, es algo complejo, pero como proceso es algo que puede reducirse a sistema, eliminando cualquier ruido - eliminando su complejidad -.

Tanto la pedagogía como las ciencias de la educación, y otras disciplinas que han trabajado sobre la explicación de la educación, han intentado reducir tal complejidad o asumirla en sentido productivo. Algunas de estas propuestas, que siguen la 
perspectiva de la Teoría de Sistemas, enfatizan la necesidad de reducir lo educativo a lo que llaman sistema educativo. Si es un sistema entonces puede planificarse, como todo sistema, y si se hace esto, entonces cada parte del sistema podrá plantearse en términos de objetivos y metas a mediano y largo plazo (SEP, 2017). Desde luego, en esta mentalidad sistémica no solo se planifica la parte institucional, se planifica igual la parte más sensible, la parte académica, la parte humana de la relación educativa. Así, en estas nuevas perspectivas se pide o se enfatiza la necesidad de la clase como algo articulado a tiempos, movimientos, tareas y productos. Mientras mejor esté planificado el proceso educativo, se evita lo que se llama ruido o complejidad, y el sistema funciona perfectamente. Sí, pero que funcione con esa perfección no significa que atienda las relaciones de singularidad de los sujetos de la educación. Tampoco significa afección en los participantes de esa relación. Si no hay afección, no hay relación educativa.

La investigación educativa ha mostrado que, aunque estemos dentro de un sistema, no somos sistema. Somos seres humanos sometidos a la lógica de la incertidumbre en cada una de nuestras acciones -incluida la acción misma de educarnos-. No podemos estar fuera del sistema educativo, porque la misma institución donde ejercemos la acción educativa está inmersa en tal sistema. Pero de eso no se sigue la factibilidad de hacer un proceso sistémico del acto educativo: hacer de la clase algo sometido a la lógica de tareas, tiempos, movimientos, acciones y productos.

Esto no significa que no se pueda objetivar lo que los estudiantes aprenden o el modo como los profesores enseñan. Pero ambas cosas tendrían formatos $\mathrm{u}$ objetivaciones, fines $\mathrm{y}$ tiempos diferentes. Ambas cosas deberían someterse a la lógica narrativa, porque es la que recupera tanto la complejidad como la incertidumbre y la única que haría posible distinguir entre el tiempo de la enseñanza y el del aprendizaje, que no tienen ninguna relación causal. Que haya una relación de enseñanza solo significa que puede ocurrir o no el aprendizaje, porque eso es algo casual, no causal. Esto implica someter a crítica la idea de una educación como proceso para dejar abierta la posibilidad de entender la educación como relación. El ejemplo de la sesión planteada al inicio es un ejemplo de educación como relación. Se esperaban preguntas, razones y argumentos, pero nunca se supo cuáles serían en concreto. 
Esta es la idea de situar un modelo educativo en el contexto neoliberal y cuyo resultado son procesos acotados a lo sistémico, mas al hacerlo, violentan cuerpos, tanto de quien enseña como de quien aprende. Como situación límite emerge el diálogo. Este salva a los participantes del proceso educativo. Justo esto es lo que mostramos en el trabajo dialógico de los niños, y es lo que articula nuestra discusión, porque en FpN se acepta un encuentro y la afección de los cuerpos, y con esto se acepta la incertidumbre, se la hace productiva. Aquí no se puede prever resultado alguno, solo una relación que inicia en un lugar y toma el camino que cada participante decide trazar en una relación que es lenguaje, gestos y cuerpo. En ese camino ocurrirá o no el aprendizaje, porque cada sujeto sabrá que toda idea será verdadera, aunque no sea aceptada por esa cualidad (Feyerabend, 1985, p. 69). Este es justo el aprendizaje que puede ser logrado.

\section{Conclusiones}

Si ponemos atención, podremos ver que hay tensión entre FpN y el proyecto educativo neoliberal o proyecto basado en competencias. Sin embargo, podría afirmarse que la forma de trabajo expuesta al inicio es la forma de trabajo sugerida en los nuevos programas. Eso fue visible desde el momento en que la misma discusión había situado a los niños en un contenido del nuevo programa, pero sobre todo en las habilidades de indagación exigidas para lograr este aprendizaje. Aquí trazamos la diferencia, porque el sistema educativo actual se ha reducido a la idea de aprender haciendo. "No pienses, solo debes hacer, haciendo aprendes", se consigna en muchos planteamientos educativos de este tipo. Sin embargo, FpN centra su argumento educativo en el pensar, en el desarrollo de habilidades para pensar.

¿Qué aprenden los niños al filosofar? Solo a tener una mente bien ordenada, como lo sugiere Morín (2003). Este es el mejor modo de aprender para la vida, de lograr esa meta de una educación basada en habilidades para la vida, la cual es la apuesta de FpN frente al modelo educativo neoliberal, que reduce la complejidad de la acción educativa a un elemental aprender haciendo.

Una mente bien ordenada es aquella que está tensada por la relación entre preguntar, pensar y aprender, porque solo quien hace preguntas puede pensar y quien piensa aprende. 


\section{Referencias}

Arquímedes. (1988). El método. México: Alianza Editorial.

Bachelard, G. (1953). El psicoanálisis del fuego. Buenos Aires: Editorial Schapire.

Bloom, B. (1971). Taxonomía de los objetivos de la educación: la clasificación de las metas educacionales: manuales I y II. Buenos Aires: Centro Regional de Ayuda Técnica, Agencia para el Desarrollo Internacional (A.I.D).

Echeverría, E. (2004). Filosofía para Niños. México: SM de Ediciones.

Eurydice. (2002). Key competencies. A developing concept in general compulsory education. Recuperado de http://www.eurydice.org

Feyerabend, P. (1985). ¿Porqué no Platón?. Madrid: Editorial Tecnos.

Goldman, A. (1986). Epistemology and Cognition. Cambridge: Harvard University Press.

Lipman, M. (1998). Pixie. Madrid: Ediciones de la Torre.

Lipman, M. (2004). Natasha: aprender a pensar con Vygotsky. Barcelona: Editorial Gedisa.

Lipman, M. (2016). El lugar del pensamiento en la educación. Barcelona: Ediciones Octaedro.

Mejía, N., López, M., \& Valenzuela, J. (2015). Pensamiento crítico en profesores de educación secundaria: caracterización de la competencia en instituciones antioqueñas (Colombia). Revista Latinoamericana de estudios educativos, 45(3), 138-177.

Morín, E. (2003). La mente bien ordenada. Barcelona: Editorial Seix Barral.

OCDE. (2006). PISA 2006. Marco de la evaluación. Conocimientos y habilidades en Ciencias, Matemáticas y Lectura.

Portillo-Torres, M. (2017). Educación por habilidades: perspectivas y retos para el sistema educativo. Revista Educación, 41(2), 2215-2644. http://dx.doi.org/10.15517/revedu.v41i2.21719

Roudinesco, E. (2018). ¿Por qué el psicoanálisis?. México: Ediciones Culturales Paidós. 
SEP. (2011). Planes de estudios 2011. México: SEP.

SEP. (2017). Modelo Educativo. Para la educación obligatoria. México: SEP.

Splitter, L., \& Sharp, A. (1996). La otra educación. Filosofía para Niños y la Comunidad de Indagación. Buenos Aires: Ediciones Manantial.

Vygotski, L. (2008). Pensamiento y lenguaje. Teorías del desarrollo cultural de las funciones psíquicas. Madrid: Aguilar. 